Popularity, theory of mind and interaction in experiments: Meta-regression

\author{
Akihito Imai \\ Graduate School of Human Development and Environment, Kobe University, Kobe, Hyogo, \\ Japan \\ Email address: 154d431d@stu.kobe-u.ac.jp
}




\title{
Popularity, theory of mind and interaction in experiments: Meta-regression
}

\author{
Abstract \\ This study aims at investigating how interaction in experiments affects effect size in studies \\ on an association between Theory of Mind and popularity. The analysis included 1946 \\ children from 17 studies. Meta-regression was employed to compare effect sizes of an \\ interaction group with that of non-interaction. Compared with an interaction group, a \\ non-interaction group are more likely to have a small effect size, indicating that researchers \\ drop hints about what answer is needed in the experiments. The result is consistent, when \\ adjusting for gender and when conducting a permutation test. The result indicates that \\ interaction with researchers in experiments could have an impact on Theory of Mind \\ studies.
}

\section{Keywords}

Theory of mind, popularity, meta-regression, meta-analysis 


\section{Introduction}

Children with an advanced theory of mind (ToM) appear to be popular in the classroom [1]. However, it is possible that, without any ToM ability, children can understand what answer is needed. Children are able to infer others' thoughts from cues like facial expressions [2].

Although these cues are seen in practical situations, especially in the classroom, it is a kind of background noise in experiments and not the ability of ToM in the light of the classic ToM definition - systematic inferences of other people's unobservable mental state [3]. To avoid direct feedback, interaction with other people in experiments should be excluded, as faux pas tasks were once conducted by using an audiocassette [4].

Many ToM studies are however implemented directly by the experimenter. The experimenter, while hoping children may answer properly, probably gives children unintended cues causing them to ace ToM experiments. This has led to suggestions that there is room for recognizing some noise — interaction with researchers in experiments in traditional ToM studies.

When children can read others' mind more accurately in any way, they are more likely to be popular in the classroom. As a result, this may have caused overestimation of effect sizes. An association between ToM and popularity may be biased, when cues are not accounted for in the experiment. 
To deal with this problem, this study compares studies assessing ToM by computer with those assessing ToM in person because there is no room for overestimation when ToM is assessed by computer. This study uses meta-regression to estimate the actual difference in effect size between these studies. Meta-regression is an extended method of meta-analysis and widely used to investigate heterogeneity between studies. In this way, this study aims at reexamining an association between ToM and popularity in a new way.

\section{Methods}

\section{Study selection}

The electronic databases, Psych INFO and Google Scholar were searched for relevant articles in March 2017. Search terms were as follows: "popularity," "sociometric," "peer acceptance," "peer likability," "peer rejection," "peer status," "peer evaluation," "peer nomination," "peer relations," "ToM," "mindreading," "mentalizing," "false belief," "mental representations," "mind understanding," and "mental states" as used in a previous

meta-analysis [1]. The Japanese database, J-STAGE, was also used to collect articles in Japanese. Citation search was also conducted.

The following inclusion criteria were used (criteria (1) to (3) followed previous meta-analysis [1]):

(1) Only healthy preschool or school-aged children under 10 years could participate. 
(2) ToM had to be assessed by more than one of false-belief understanding, hidden emotion, affective perspective-taking, or faux pas tasks.

(3) Sociometric or perceived popularity had to be assessed by a peer or a teacher.

(4) Effect size(s), $N$ and gender ratio must be reported or convertible. An association between ToM and popularity was evaluated by Pearson correlation coefficient or $r$. If multiple coefficients were reported in a study, these were synthesized into one coefficient unless a study has multiple age groups.

(5) Measures of ToM have to be identifiable (computer or person). Coding is organized based on whether ToM was assessed in person (e.g., researcher and experimenter) or by computer. Studies assessing ToM in person are categorized as an interaction group and those assessing ToM by computer are categorized as a non-interaction group.

(6) Peer-reviewed articles were from publication in either English or Japanese.

\section{Statistical analysis}

This study uses random-effects meta-regression. Meta-regression can be seen as an extension to standard meta-analysis, and is rigorous as well as effective. The statistical software Stata 15.0 was used for all data analysis. The meta-regression algorithm was implemented by using version 2.6 .1 of the metareg command [5]. Publication bias was assessed by using version 4.1 .0 of the metabias command [6]. 


\section{Result}

According to criteria, 17 studies (22 effect sizes) were identified. Detailed characteristics were coded for each study and presented in Table 1. As shown, a total of 1946 children were included.

The result of meta-regression, without fitting any covariates, showed that effect size was associated with interaction in the experiments, $\beta=.134(95 \% \mathrm{CI} .006-.262), p=.041$, and between-study heterogeneity $\left(I^{2}\right.$ res $)$ was $27.68 \%$. After controlling for gender, the result was still consistent, $\beta=.137(95 \%$ CI $.003-.272), p=.046, I_{\text {res }}^{2}=30.82 \%$.

To overcome the small sample size which increases the chance of a false-positive (type I) error, permutation analysis was implemented. The result was marginally significant ( $p$ $=.083$ ), indicating consistency with previous analyses. The result of Egger's test showed that publication bias was non-significant $(p=.735)$. Taken together, these results indicate rejection of the null hypothesis - effect of interaction with researchers in experiments was zero. 


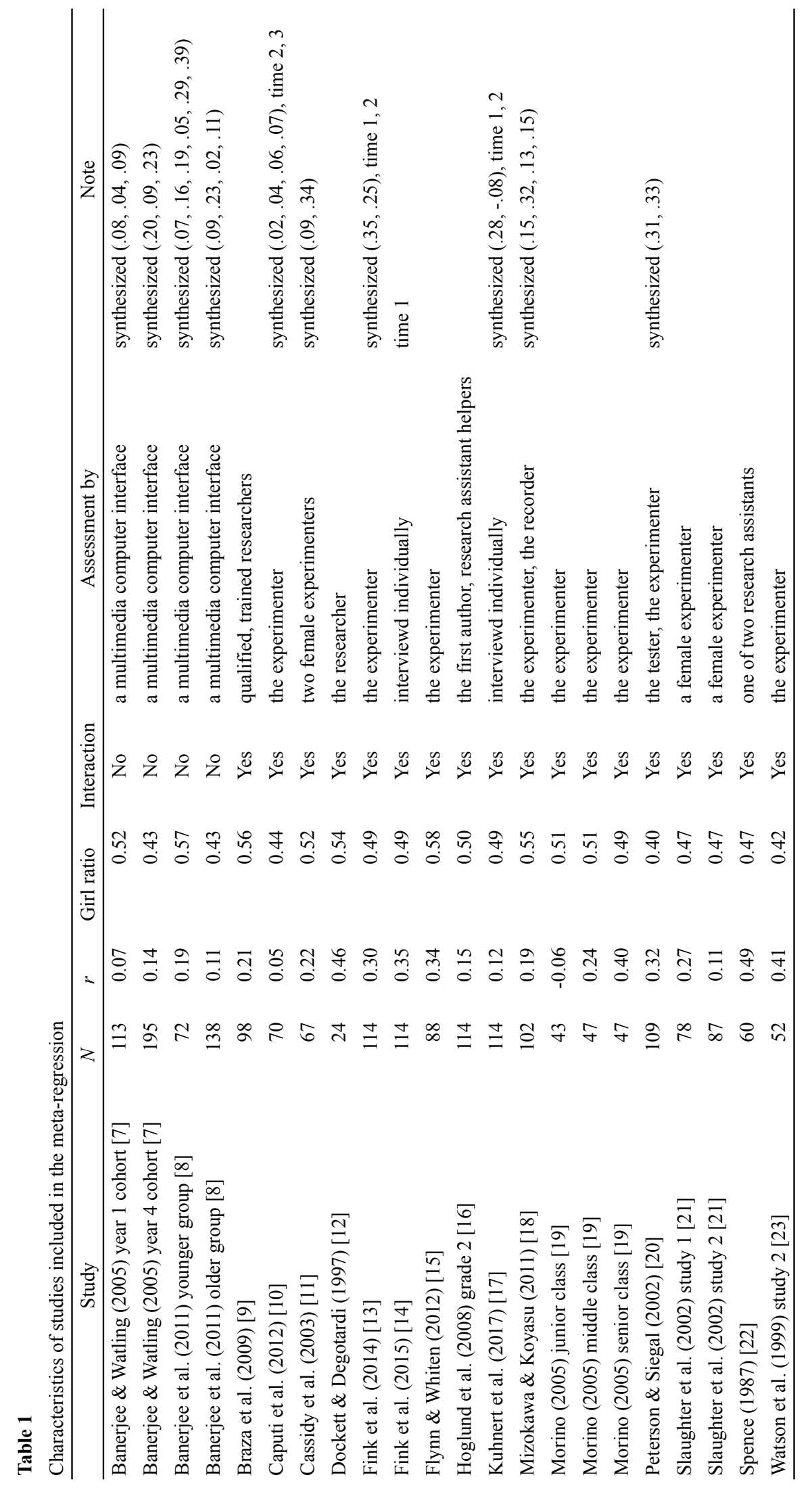




\section{Discussion}

Using data from 17 studies, this study found evidence for a disparity between interaction and non-interaction groups in studies of ToM and popularity.

The result seems to be open to interpretation. On the one hand, the result indicates that there seems to be a cue from the experimenter to obtain a wanted answer in traditional ToM studies. On the other, the result can lead to suggestion that tasks with non-interaction can underestimate children's real ToM abilities because real life is full of social cues and signals.

One limitation of this study is that the total number of included effect sizes was limited. Most studies included in this analysis had relatively small sample sizes. The results of this analysis should therefore be interpreted with some caution. In addition, like any meta-analysis, this study cannot account for unmeasured variables such as the personal characteristics of experimenters. Some experimenters can control their cues and others cannot. Unmeasured variables could have had an impact on this analysis.

This study highlights that interaction in experiments indicates overestimation in traditional study design. The result indicates that experimenters are, on average, likely to give children

cues to answer properly. It seems that this problem is difficult to deal with, because this interaction is due to traditional ToM experiment design. Recommended methods to 
overcome this problem can be ToM assessment by using audiocassette [4], computer [7, 8] or booklet [24].

\section{Data availability}

Supplementary materials can be found at https://osf.io/82auq/.

\section{References}

[1] Slaughter V. Imuta K. Peterson CC. Henry JD. Meta-analysis of theory of mind and peer popularity in the preschool and early school years, Child Dev. 86 (2015) 1159-1174. doi:10.1111/cdev.12372

[2] Baron-Cohen S. Wheelwright S. Jolliffe AT. Is there a "language of the eyes"?: evidence from normal adults, and adults with autism or Asperger syndrome, Vis. Cogn. 4 (1997) 311-331. doi:10.1080/713756761

[3] Premack D. Woodruff G. Does the chimpanzee have a theory of mind?, Behav. and Brain Sci. 1 (1978) 515-526. doi:10.1017/S0140525X00076512

[4] Baron-Cohen S, O'riordan M, Stone V, Jones R, Plaisted K. Recognition of faux pas by normally developing children and children with Asperger syndrome or high-functioning autism. J. of Autism and Dev. Disorders. 29 (1999) 407-418. doi: 10.1023/A:1023035012436

[5] Harbord RM. Higgins JP. Meta-regression in Stata, Stata J. 8 (2008) 493-519. 
[6] Harbord RM. Harris RJ. Sterne JA. Updated tests for small-study effects in meta-analyses, Stata J. 9 (2009) 197-210.

[7] Banerjee R. Watling D. Children's understanding of faux pas, Hell. J. of Psychol. 2 (2005) 27-45.

[8] Banerjee R. Watling D. Caputi M. Peer relations and the understanding of faux pas: longitudinal evidence for bidirectional associations, Child Dev. 82 (2011) 1887-1905. doi:10.1111/j.1467-8624.2011.01669.x

[9] Braza F. Azurmendi A. Munoz JM. Carreras MR. Braza P. García A. Sorozabal A. Sánchez-Martín JR. Social cognitive predictors of peer acceptance at age 5 and the moderating effects of gender, Br. J. of Dev. Psychol. 27 (2009) 703-716. doi:10.1348/026151008X360666

[10] Caputi M. Lecce S. Pagnin. A. Banerjee R. Longitudinal effects of theory of mind on later peer relations: the role of prosocial behavior, Dev. Psychol. 48 (2012) 257-270. doi:10.1037/a0025402

[11] Cassidy KW. Werner RS. Rourke M. Zubernis LS. Balaraman G. The relationship between psychological understanding and positive social behaviors, Soc. Dev. 12 (2003) 198-221. doi:10.1111/1467-9507.00229

[12] Dockett S. Degotardi S. Some implications of popularity at age four, J. of Aust. Res. in Early Child. Educ. 1 (1997) 21-31.

[13] Fink E. Begeer S. Hunt C. Rosnay M. False-belief understanding and social preference over the first 2 years of school: a longitudinal study, Child Dev. 85 (2014) 2389-2403. doi:10.1111/cdev.12302 
[14] Fink E. Begeer S. Peterson CC. Slaughter V. Rosnay M. Friendlessness and theory of mind: a prospective longitudinal study, Br. J. of Dev. Psychol. 33 (2015) 1-17. doi:10.1111/bjdp.12060

[15] Flynn E. Whiten A. Experimental "microcultures" in young children: identifying biographic, cognitive, and social predictors of information transmission, Child Dev. 83 (2012) 911-925. doi:10.1111/j.1467-8624.2012.01747.x

[16] Hoglund WL. Lalonde CE. Leadbeater BJ. Social-cognitive competence, peer rejection and neglect, and behavioral and emotional problems in middle childhood, Soc. Dev. 17 (2008) 528-553. doi:10.1111/j.1467-9507.2007.00449.x

[17] Kuhnert RL. Begeer S. Fink E. de Rosnay M. Gender-differentiated effects of theory of mind, emotion understanding, and social preference on prosocial behavior development: a longitudinal study, J. of Exp. Child Psychol. 154 (2017) 13-27. doi:10.1016/j.jecp.2016.10.001

[18] Mizokawa A. Koyasu M. Understanding false beliefs, hidden emotions, and social interactions among five- and six-year olds, Jpn. J. of Dev. Psychol 22 (2011) 168-178. doi:10.11201/jjdp.22.168

[19] Morino M. Preschoolers' theory of mind, understanding of emotions, and interactions with peers, Jpn J. of Dev. Psychol. 16 (2005) 36-45. doi:10.11201/jjdp.16.36

[20] Peterson CC. Siegal M. Mindreading and moral awareness in popular and rejected preschoolers, Br. J. of Dev. Psychol. $20 \quad$ (2002) 205-224. doi:10.1348/026151002166415 
[21] Slaughter V. Dennis MJ. Pritchard M. Theory of mind and peer acceptance in preschool children, Br. J. of Dev. Psychol. 20 (2002) 545-564. doi:10.1348/026151002760390945

[22] Spence SH. The relationship between social-cognitive skills and peer sociometric $\begin{array}{lllllll}\text { status, } & \text { Br. J. } & \text { of } & \text { Dev. Psychol. } 5 & 5 & \text { (1987) }\end{array}$ doi:10.1111/j.2044-835X.1987.tb01071.x

[23] Watson AC. Nixon CL. Wilson A. Capage L. Social interaction skills and theory of mind in young children, Dev. Psychol. 35 (1999) 386-391. doi:10.1037/0012-1649.35.2.386

[24] Hayashi H. Children's moral judgments of commission and omission based on their understanding of second-order mental states, Jpn. Psychol Res. 49 (2007) 261-274. doi:10.1111./j.1468-5884.2007.00352.x 